\title{
General Subject Index
}

absolute chronology 59, 60, 304

Adam 226, 254, 264, 314, 331

agrarian expansion 4, 313, 315

agriculture, see also crops; livestock

agricultural development 316

agricultural economy 308-318

agricultural labour 277, 308-309

agricultural methods 314,316

agricultural production 275, 280, 309, 314, 316-317

commercialisation 308

diversification 252, 258, 277, 280, 308

household-based processing 277, 308-309, 314

intensification 247, 258, 277, 280, 308-311, 315

subsistence 310

Ajanta Caves 61

Alampur 57

Amaravati 17, 234, 305

animals, see also bone; livestock

bird 233, 283, 288

blackbuck 283

cattle 283, 285-289, 291, 292-295, 311

crocodile 283, 289

deer 283

dog 281-282, 283, 289, 309, 311

fish 283, 285, 288

goat 281-290, 292, 293, 309-312

horse 283

jackal 283

monkey 283

pig 281-282, 284-295, 309-312

sheep 281-290, 292, 293, 309-312

turtle 283

water buffalo 283-284

zebu 283

Annales school 326

Archaeological Survey of India 6, 8, 10, 324, 328, 329

area excavation 10, 62, 69, 329

Arrian 13

arrowheads 87, 185-187, 233

Arthashastra 311, 313

Asaf Jahi period 303

Aśmaka 11-12, 15, 16

Asoka 15

Assaka 11

axles 193

Bahmani Sultanate 228

Ahmed Shah II 228

balls 193, 196, 198, 201

bangles 84, 158, 171-179, 232, 233, 242

Bāvari 11

beads $19,84,87,158,160-171,232,242,333$

Berenike 273, 274, 280

Bhadrabahu 12

Brahmanic texts 12-13

Mahabharata 11, 12

Purānas 12, 13

\author{
BrahmaPurāṇa 12, 13 \\ LingaPurāna 12 \\ MatsyaPurāna 12 \\ PadmaPurāna 12
}

Bharuch 12, 13

Bhitargaon 36, 61, 306

Bhitari 58, 61, 304

Bhojakas 15

Bhokardan 18, 57, 101, 112, 115, 116, 118, 119, 125, 129, 139, 227, $242,254,264,284,313,319,320$

Bhumara 61

Bhuvaneshvara 62

Bodhgaya 61, 306

bone $71,87,183,194,233,242,281-295$

bone objects $87,183,194,233,242$

faunal remains 281-295, 309

age at death 289

Minimum Animal Units 286-287, 288, 290-291

Minimum Number of Individuals 286

Number of Identified Specimens 282-283, 285-287

human remains 71

borers 185,188

bowls 105, 112, 115, 118, 119, 122, 127, 129, 131, 133, 136, 185, 194, 196-198, 199

box trenches 10, 329

Brahmagiri 57, 93

Brahmapuri 14, 18, 93, 106, 107, 115, 118, 124, 125, 139, 164

Brahminism 3, 4, 16, 57-58, 305, 313-317, 333-334, 335

brass 184

brick drain 19, 300-302

British period 3, 97, 225, 303

bronze 184-185

buckles 185, 193

Buddhism 314, 315

Buddhist texts 11-12

Anguttara Nikāya 11, 12

Jatakas 11, 313

Assaka Jataka 11

Baveru Jataka 11

Chulla Kalinga Jataka 11

Mahabharata 11, 12

Mahāmayurī 12

Sutta Nipāta 11, 12

butchery 288

$\mathrm{C}^{14}$ dates $95,96,106$

cereals $246-247,252,253-263,275,280$

barley $246,247,253,254,256,257,258-259,313$

emmer 258

millet 247, 248-249, 253, 255, 256-257, 259-263, 280, 308, $309,313,314,315,317$

rice $246,247,253,254,255-258,280,313,314-315$

sorghum 252, 253, 254, 256, 259-261, 280, 313

wheat $6,246,247,253,254,258-259,313,314$

Chakradhara 14

Chalcolithic 158, 234, 245, 247, 255, 258, 260, 262, 265, 266, 267, 276, 277, 278, 279, 309 
Chalukyan architecture 57

Chammak 334

Charsadda 329

chisels 185, 187-188, 233

Chejerla 60, 61

coins, see also hoards; monetization

British coins 19, 225

coin circulation 3, 91, 96, 97, 318-320

coin deposition 96, 97, 229-231, 302, 308, 318-320

'elephant'-type 219, 221, 227, 235, 237

'hollow cross'-type 96, 215-217, 225, 226, 235, 300

Indo-Sasanian coins 228, 238

Islamic coins 225, 228

Kshatrapa coins 221, 227, 228, 231, 232, 238

Maitraka coins 62, 228, 231

post-Satavahana coins 97, 221, 225, 228, 231, 319

pre-Satavahana coins 95, 96, 215-217, 226-227, 231, 232, 300,

319

punch-marked coins $96,226,234-235,300$

Sarvva-Bhattaraka coins 62, 228, 231

Satavahana coins 19, 20, 91, 95, 96, 219-221, 223, 227-228,

231, 232, 237-238, 239, 319

'tree'-type 217, 226-227

copper 19, 171-174, 179-180, 182, 184-185

copper-plate inscriptions $3,15-16,315,316,331-334$

cotton $6,13,270-274,280,308,309,317$

cowrie shells $285,288,319-320$

crops, see also agriculture; cereals; cotton; flax; pulses

cash-cropping 274, 275, 308, 310

crop-livestock interaction 309, 312

cropping strategies 280, 308, 309, 312, 314, 315

crop-processing 246, 260, 276, 277, 309

crop residues 309,310

double-cropping 247, 260, 280, 308, 310, 315

fallow periods $247,280,308$

summer crops $247,253,257,268$

surplus $274,308,310,313,314$

winter crops 246, 247, 253, 258, 263, 267-268, 275-276, 280, 308, 314, 315

dabbers 199

dairy products 289

Darra 61

Daulatabad 14, 228

Delhi school 325, 326

Deogarh 61, 306

Dhanyakataka 17

Dnyaneshwara 14

ear-studs 180, 181, 233, 242

eggplant 268

Eknath 14

Elephanta Caves 61

Eran 326, 327

Fa-Hsien 311

fasteners 184,191

feudalism 3, 316, 325, 326

figurines $15,19,56,108,204,205-213,239,241,242,307$

flax 274-275 flotation 5, 10, 244, 245-246, 255, 280

fodder $255,270,276,278$

folklore $11,14-15,17$

gahapati 314, 317

glass $165-167,174-176,180,182,194,213,214,232,233,242,321$

Godavari river 4-6, 8, 9, 11-14, 17, 19, 24, 244, 280, 314-316, 335

gold 182

Gunāđhya 13

Gupta period 3, 228, 313, 316, 317, 318, 320, 325

Gupta architecture 58, 61

Gupta coins 228, 238

Kumara Gupta 228, 238

Harappa 263, 269, 270-272, 276, 329

Harappan civilization 112, 256, 258, 264-265, 267, 268, 269, 270, 275,276

hemp 275-276

Hinduization 308, 316

historiography $3,312,324-328$

hoards 318,320

Amaravati hoard 234

Jogalthembi hoard 228

Wategaon hoard 227

Iron Age 254-257, 261, 264-266, 268, 277, 279, 333, 334

iron objects 19, 185-193, 196, 233, 321

iron slag 213

irrigation 6, 247, 258, 274, 308, 313-316

Isanasivagurudevapaddhati 57-58

ivory 19, 176-177, 242, 321

Jaggayyapeta 305

Jaina texts 12

Āvaśyakaniryukti 12

Ḅ̣hatkalpabhāṣya 12

Gacchācārṿrtti 12

Kālakāchārya Kathānaka 12

Kalpasūtra 12

Nišîthacūrṇi 12

Pinḍadaniryukti 12

Tiloya-Paṇnati 12

Vasudeva Hindi 12

Junnar 314

Kanheri 15, 314

kaolin 19, 56, 205, 208, 233, 239, 242, 307

knives 187, 233, 283

kohlsticks 19, 84, 182, 242

Kshatrapas 14, 15, 221, 227, 228, 231, 232, 238

Chashtana 14

Nahapana 12, 227, 228, 238

Kuda 14, 17

Kunda 61

lamps 198, 199

land ownership 314, 316

endowments 313, 315-317, 333, 335

grants 3, 4, 314-316, 324, 331-333

Laws of Manu 309, 311 
lead objects 193

Limbārāmikā 16

lime mortar 28, 43, 44, 56, 67, 70, 71

livestock, see also animals; bone

age at death 289

cattle 283, 285-289, 291, 292-295, 311

crop-livestock interaction 309, 312

goat 281-290, 292, 293, 309-312

pig 281-282, 284-295, 309-312

sheep 281-290, 292, 293, 309-312

transhumant migration 310

Mahānubhāva literature 14

Leelā-Charitra 14

Smrtisthala 14

Sthānpothī 14

Maheshwar 3, 93, 101, 107, 111-113, 115, 116, 118, 119, 125, 129, 131, $136,139,141,226,319,320$

Mahurjhari 333, 335

Mandhal 331

Marathi literature 14

Marxist perspectives 3, 316, 325-326

Mauryan period 3, 6, 15, 107, 300, 313

Megalithic 256, 271, 333

microliths 92, 158, 159, 300

millet 247, 248-249, 253, 255, 256-257, 259-263, 277, 278, 279,

$280,308,309,313,314,315,317$

African millets 247, 255, 259-261, 308, 309, 314, 317

broomcorn millet 261-262

browntop millet 262-263

Brachiaria ramosa 247, 248, 252, 254-257, 260-262, 280

Chinese millets 261-262

foxtail millet 261-262, 280

finger millet 259-261

Indian small millets 262-263

kodo millet 247, 254-257, 262, 263, 277, 280, 308

pearl millet 253, 256-257, 259-261, 280

sawa millet 262-263

Mohenjo-daro 270, 271, 329

monetization 318-320

Mughal period 19, 84, 87, 225, 228, 303, 309

Aurangzeb 225, 228

Mūlaka 11, 12, 15, 16

Murundas 12

Nachana 61

Naga 12-15

Nagara 18, 107, 304

Nagarjunakonda 305

Nasik 15-17, 18, 93, 105, 107, 115, 116, 118, 119, 122, 125, 133, 139, $141,163,167,182,194,281,284,304,314$

Neolithic 245, 247, 253, 255, 256, 258, 259, 262-268, 271, 277-279

Nevasa 18, 58, 90, 93, 95, 101, 105-108, 111-113, 115, 116, 118, 119, $122,124,125,129,131,139,177,179,182,194,219,227,234$, 235, 237, 254, 257, 264, 271, 275, 281, 284, 304, 313, 319321

Pattadakal 57

Periplus Maris Erythraei 11, 13, 274, 309, 321

Petenikas 15

Pitalkhora Caves 15
Pliny 13

population growth $280,313,314,317$

porcelain $92,97,194,204,213$

pottery $4,19-20,32,57,60,62-65,70,73,81,84,86,87,90-93$, 95, 97, 101-157, 199, 233, 234, 320-321, 323

Black and Red ware (B\&R) 91-92, 95, 101-102, 104-105,

112-113, 119, 131, 201, 204, 300

Black Burnished ware (BBW) 112, 119, 127, 131, 137

Blue and White Frit (PERS) 93, 97, 108-110, 111, 149

Chinese Blue and White porcelain (CBW) 93, 108, 110

Jorwe ware 92, 96, 105-106, 119, 234, 300

Northern Black Polished ware (NBP) 19, 91-93, 95, 106-107,

113, 149, 300

Red Polished ware (RPW) 84, 91-93, 95, 107-108, 110, 113, 149, 234

Slip-painted ware (SLIP) 93, 110, 111, 149

Thick Grey ware (GREY) 73, 84, 85, 91, 92, 95, 97, 104, 111-113, 122, 125, 133, 139

White and Red Painted (WARP) 105, 110, 111, 136, 149

Prakash 18, 101, 105, 107, 108, 111-113, 115, 116, 118, 119, 133, 139, 141, 226

Prakrit literature 13

Bṛhatkathā 13

Brhatkathā-Manjirī 13

Gāthā Saptaśati 13

Kathā-Saritsāgara 13, 16

Ptolemy 11, 13-14, 16-17

pulses $6,246-247,252,257,258,263-268,280,308,315$

chickpea 263, 267, 268, 308

cowpea 266

grasspea $263,267,308$

horsegram 266-267

hyacinth bean 267

lentil 263, 266-268, 308

moth bean $265-266$

mung bean $264-265$

pigeonpea 267

urd bean 264-265

winter pulses $246-247,257,258,267-268,280,308,315$

querns $19,87,198,242$

Qutb Shahi Sultanate 225, 228

Raika 310

Rajim 62

Ramtek 33, 60, 62

Rashtrakutas 16, 19-20, 57, 302, 304, 315, 316

Govind III 16

Indra III 16

Rathikas 15

rings $179-180,191,242$

finger-rings $179-180,242$

toe-rings 180

ring-wells 84-85, 301

rivets 184,191

Roman trade 274, 280, 321

Roman amphorae 95, 108, 152, 321

rural economy 312-314, 323 
safflower 275

Sakor 61

Sanchi 18, 314, 315, 328, 329

sangha 314

Satavahanas 6, 12-17, 19-20, 88-89, 91, 95, 96, 219-221, 227-228, 231, 242, 237-238, 280, 302, 304, 311-312, 313-316, 320

Gautamiputra Yajnasri Satakarni 237-239

Gotamiputa Siri Satakani 15-17, 96, 227-228, 238

Hāla 13

Kosikiputa Siri Satakani 219, 227

Sālivāhana 12, 14-16

Vasithiputa Siri Pulumavi 13, 15, 227-228, 237

Satdhara 328

sati $326-327$

Satpura hills 331

sculpture 56, 213, 224, 305, 307, 334

seals and sealings $16,84,232,242$

sesame $268-269$

shell 168, 177-179, 180, 213, 232, 242, 283, 288, 319-320

Sirpur 62

skin rubbers 84, 199, 233, 242

Strabo 13

subaltern studies 326-327

Sufism 14, 228

Taxila 93, 226, 329

temples 23-80, 304-308

Buddhist cave temples 15

brick temples at Paithan 5-6, 23-80, 92-93, 95-97, 149,

304-308

1937 excavation 19, 24, 27, 41

1965 excavation and restoration 19, 27

coin deposition 229-231

faunal remains $288-289$

early Hindu temple architecture 23, 307-308

adhisthana mouldings 33, 61-62, 306, 307

foundations $56-58,305$

garbhagriha 61, 304

\section{kumuda 61}

mandapa 62, 304, 306-308

mulaprasada 62, 305-306

shikhara 61, 306, 307

shrine 305-307

standard temple plan 304-305

triratha 58, 61, 306

economic and political role 3-4, 313, 315-317, 324, 325-326, 333, 335

Ter 13, 18, 62, 107, 115, 116, 118, 119, 122, 125, 129, 139, 141, 205 , 242, 274, 309, 313

terracotta $14-15,19,56,84-85,160,169-171,179,180-182$, 198-201, 205-214, 232-233, 234, 238-242, 307

textile production $270-274,280,308-309$

Tigawa 61

toys $14-15,201-205,233$

typology 102, 113, 139, 149

Ujjain 14, 15, 274

urban archaeology 98, 329

urbanization 314

Varkari 14

Vakatakas 15, 95, 304, 313, 315, 318, 331-334

Pravarasena II 15, 334

vases 198, 199, 234

Veerapuram 254, 256, 264, 305

Vidarbha 14, 17, 226, 331-335

Vidisha 314-316

Vikrama 14, 15

weeds $246,252,253,262-263,276-279,309,314,317$

Wheeler method 329

wire 185, 193

Yadava period 14

Yelleshvarum 305 
